\title{
ac Response in the Nonequilibrium Anderson Impurity Model
}

\author{
Tai-Kai $\mathrm{Ng}$ \\ Department of Physics, Hong Kong University of Science and Technology, Clear Water Bay Road, Kowloon, Hong Kong
}

(Received 5 July 1995)

\begin{abstract}
The ac response of a single Anderson impurity coupled to two reservoirs with different chemical potentials is studied using an equation of motion approach. In the Kondo regime, we show that resonant behavior in the ac response is found in the low-frequency limit and the frequency of the ac field matches the chemical potential differences. The resonant behavior is a direct consequence of the "double" Kondo peak structure in the one-electron spectral function.

PACS numbers: 72.15.Qm, 73.20.Dx, 73.50.Fq
\end{abstract}

Recently there has been a lot of interest in the study of electronic transport through quantum dot devices or Anderson impurity at low temperature, especially in the nonlinear regime where the difference in chemical potentials $\Delta \mu$ between the two reservoirs coupling to the device (or impurity) is large [1,2]. The problem of Anderson impurity in a nonequilibrium environment has been studied by different groups using different techniques, both in the single channel [3-5] and in the two channel case [6]. In particular, based on large- $N$ type theories, it has been suggested that a Kondo type effect can survive in a nonequilibrium system at low temperature [4-6]. As a result of the Kondo effect, it was predicted that (1) a resonant peak will be found in the $d I / d V$ vs $V$ curve, pinned at $V=0$, where $I$ is the current and $V$ is the voltage difference between the reservoirs, and (2) the impurity one-electron Green's function will exhibit a two-peak structure, with the peak position pinned at the two Fermi surfaces. The magnitude of the peak decreases gradually as the voltage difference $V$ increases, because of inelastic scattering associated with finite chemical potential differences. Experimentally, the resonant peak in the $d I / d V$ vs $V$ curve has been observed [2] in $I-V$ measurements. However, the more subtle twopeak structure in the electron spectral function cannot be probed by (dc) $I-V$ measurements, and thus has not been confirmed. In this paper we show that the two-peak structure in the electron spectral function can be probed by imposing on top of the dc bias an ac bias voltage. The double-peak structure in the electron spectral function gives rise to resonant behaviors in the current response as the ac frequency matches the dc bias voltage, and can be observed directly in experiment.

Phenomenologically, the resonant behavior can be understood very crudely using a lowest order Fermi golden argument: the ac current response to an applied ac voltage with frequency $\omega_{0}$ is proportional to the transition rate exciting an electron with energy $\epsilon$ below the Fermi surface to a state with energy $\epsilon+\hbar \omega_{0}$ above the Fermi surface. Using the Fermi golden rule, the transition rate is proportional to the single-electron joint density of states $\rho(\epsilon) \rho\left(\epsilon+\hbar \omega_{0}\right)$ and may exhibit a peak when the joint density of states has a maximum, depending on the transition matrix element. This may happen in the nonequilibrium Anderson impurity problem when the frequency $\omega_{0}$ goes to zero and when it matches the voltage differences between the two reservoirs because of the Kondo peaks pinned at the two Fermi surfaces. To be more precise, we shall compute in the following the linear response current of a nonequilibrium Anderson impurity to an ac bias voltage in the Kondo regime. The Kondo effect will be treated in an equation of motion approach [4] which can be generalized quite easily to include ac perturbation. The ac effect will be treated following the formulation employed by Wingreen, Jauho, and Meir [7] in studying time-dependent transports in noninteracting resonant tunneling systems.

Following Wingreen, Jauho, and Meir we consider the following time-dependent Hamiltonian,

$$
\begin{aligned}
H= & \sum_{k, \eta, \sigma} \epsilon_{k \eta}(t) c_{k \eta \sigma}^{\dagger} c_{k \eta \sigma}+\sum_{k, \eta, \sigma}\left[t_{\eta} c_{k \eta \sigma}^{\dagger} d_{\sigma}+\text { H.c. }\right] \\
& +\sum_{\sigma} \epsilon_{0}(t) d_{\sigma}^{\dagger} d_{\sigma}+U n_{d \uparrow} n_{d \downarrow}
\end{aligned}
$$

where $\eta=L, R$ are Fermi sea indices, $c_{k \eta \sigma}^{\dagger}\left(c_{k \eta \sigma}\right)$ are creation (annihilation) operators for electrons in reservoir $\eta$ and with spin $\sigma . d_{\sigma}^{\dagger}\left(d_{\sigma}\right)$ are electron creation (annihilation) operators on the Anderson impurity. The last term in $H$ represents the Coulomb interaction between electrons on Anderson impurity where $n_{d \sigma}$ is the impurity (spin- $\sigma$ ) electron occupation number operator. Electronic states in the left and right reservoirs are filled up to chemical potentials $\mu_{L}$ and $\mu_{R}$, respectively, where $\mu_{L} \neq$ $\mu_{R}$ in general (dc bias). The ac bias is reflected in the time-dependent single-particle energies $\epsilon_{k \eta}(t)$ and $\epsilon_{0}(t)$. In particular, we consider

$$
\begin{gathered}
\epsilon_{k \eta}(t)=\epsilon_{k}+V_{\eta} \cos \left(\omega_{0} t\right), \\
\epsilon_{0}(t)=\epsilon_{0}+V_{0} \cos \left(\omega_{0} t\right) .
\end{gathered}
$$

The time-dependent current flowing out of reservoir $\eta$ to the Anderson impurity can be written as [7]

$$
\begin{aligned}
J_{\eta}(t)= & -\frac{2 e}{\hbar} \int_{-\infty}^{t} d t^{\prime} \int \frac{d \epsilon}{2 \pi} \operatorname{Im}\left\{e^{i \epsilon\left(t-t^{\prime}\right)} \Gamma^{\eta}\left(t^{\prime}, t\right)\right. \\
& \left.\times\left[G_{d}^{<}\left(t, t^{\prime}\right)+n_{\eta}(\epsilon) G_{d}^{r}\left(t, t^{\prime}\right)\right]\right\},
\end{aligned}
$$


where $\Gamma^{\eta}\left(t^{\prime}, t\right)=2 \pi \rho(0)\left|t_{\eta}\right|^{2} e^{i \int_{t^{\prime}}^{t} d t^{\prime \prime} V_{\eta} \cos \left(\omega_{0} t^{\prime \prime}\right)}$, with $\rho(0)$ the density of states in the two reservoirs. We have assumed that the density of states is independent of energy and is the same for the two reservoirs in writing down the above expression. Thus to compute the time-dependent current, we have to compute the impurity electron retarded Green's function $G_{d}^{r}\left(t, t^{\prime}\right)$ and Keldysh Green's function $G_{d}^{<}\left(t, t^{\prime}\right)$, in the presence of interaction $U$ and the ac voltage fluctuation $V_{\eta}\left(V_{0}\right) \cos \left(\omega_{0} t\right)$. We shall first consider the retarded Green's function $G_{d}^{r}\left(t, t^{\prime}\right)$.

In the presence of both interaction and ac voltage, it is difficult to compute $G_{d}^{r}\left(t, t^{\prime}\right)$ accurately. In the following, we shall consider $G_{d}^{r}\left(t, t^{\prime}\right)$ in the equation of motion approach. The approach is not quantitatively reliable in describing detail features of (nonequilibrium) Kondo resonances. However, it gives correct qualitative features in the case with dc bias voltage [4] and has the advantage that it can be easily extended to the case with ac bias. We use this approach because of its simplicity and also because we are interested only in qualitative features of the ac current response in this paper. The equation of motion method consists of differentiating the Green's function $G_{d}^{r}\left(t, t^{\prime}\right)$ with respect to time, thereby generating higher-order Green's functions which eventually have to be truncated to close the equation for $G_{d}^{r}\left(t, t^{\prime}\right)$. The approximation we take to truncate the equation of motion is identical to the one used by Meir, Wingreen, and Lee [8] for the Anderson impurity model without ac field. Introducing the gauge transformation

$$
G_{d}^{r}\left(t, t^{\prime}\right)=e^{-(i / \hbar) \int_{t^{\prime}}^{t} d t^{\prime \prime} V_{0} \cos \left(\omega_{0} t^{\prime \prime}\right)} \bar{G}_{d}^{r}\left(t, t^{\prime}\right)
$$

and the Fourier transform $\bar{G}\left(\omega, \omega^{\prime}\right)=\int d t \int d t^{\prime} \times$ $\bar{G}\left(t, t^{\prime}\right) e^{i \omega t} e^{i \omega^{\prime} t^{\prime}}$, we obtain in the limit $U \rightarrow \infty$, and in the Kondo limit $\epsilon_{0}(t) \ll \mu_{L}, \mu_{R}$,

$$
\begin{aligned}
\left(\hbar \omega-\epsilon_{0}\right) \bar{G}_{d}^{r}\left(\omega, \omega^{\prime}\right)= & 2 \pi \hbar \delta\left(\omega-\omega^{\prime}\right)\left(\frac{1}{2}\right) \\
& +\int \frac{d \omega^{\prime \prime}}{2 \pi} \bar{\Sigma}^{r}\left(\omega, \omega^{\prime \prime}\right) \bar{G}_{d}^{r}\left(\omega^{\prime \prime}, \omega^{\prime}\right),
\end{aligned}
$$

$\bar{\Sigma}^{r}\left(\omega, \omega^{\prime}\right)$ is the Fourier transform of the self-energy function $\bar{\Sigma}^{r}\left(t, t^{\prime}\right)$, where $\bar{\Sigma}^{r}\left(t, t^{\prime}\right)=\bar{\Sigma}_{b}^{r}\left(t, t^{\prime}\right)+\bar{\Sigma}_{i}^{r}\left(t, t^{\prime}\right)$,

$$
\bar{\Sigma}_{b}^{r}\left(t, t^{\prime}\right)=\sum_{\eta} \Gamma_{\eta} \int_{-D}^{D} \frac{d \epsilon}{2 \pi \hbar} \bar{g}_{\eta}^{r}\left(\epsilon, t, t^{\prime}\right)
$$

is the noninteracting self-energy coming from the tunneling of electrons from the impurity state to outside reservoirs [7], $D$ is the bandwidth, and $\Gamma_{\eta}=2 \pi \rho(0)\left|t_{\eta}\right|^{2}$,

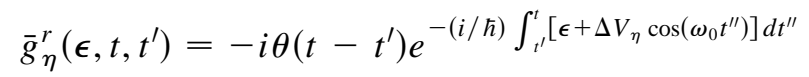

is the time-dependent retarded Green's functions in the reservoir $\eta$ in the uncoupled $\left(t_{L(R)} \rightarrow 0\right)$ limit, $\Delta V_{\eta}=$ $V_{\eta}-V_{0}$. In particular, in the limit $D \rightarrow \infty, \bar{\Sigma}_{b}^{r}\left(t, t^{\prime}\right) \rightarrow$
$-i(\Gamma / 2) \delta\left(t-t^{\prime}\right)$ where $\Gamma=\Gamma_{L}+\Gamma_{R}$, and

$$
\bar{\Sigma}_{i}^{r}\left(t, t^{\prime}\right)=\sum_{\eta} \Gamma_{\eta} \int_{-D}^{D} \frac{d \epsilon}{2 \pi \hbar} n_{\eta}(\epsilon) \bar{g}_{\eta}^{r}\left(\epsilon, t, t^{\prime}\right)
$$

is the interaction self-energy evaluated in the limit $U \rightarrow \infty$ $[4,8]$. The factor $(1 / 2)$ in Eq. (3a) originates from the factor $1-\left\langle n_{d-\sigma}\right\rangle$, which goes to $1 / 2$ in the Kondo limit $[4,8]$. A similar result is obtained for the advanced Green's function $\bar{G}_{d}^{a}\left(\omega, \omega^{\prime}\right)$ with $\bar{g}^{r}$ replaced by $\bar{g}^{a}$ in the self-energy function $\overline{\bar{\Sigma}}^{a}\left(t, t^{\prime}\right)$.

The Green's function $G_{d}^{<}\left(t, t^{\prime}\right)$ cannot be obtained straightforwardly from the equation of motion approach without introducing additional assumptions. In the following we shall obtain $G^{<}$in an alternative approach. Using the formal Keldysh Green's function technique, it can be shown that [7] $\bar{G}_{d}^{<}\left(t, t^{\prime}\right)=$ $\int d t_{1} \int d t_{2} \bar{G}_{d}^{r}\left(t, t_{1}\right) \bar{\Sigma}^{<}\left(t_{1}, t_{2}\right) \bar{G}_{d}^{a}\left(t_{2}, t^{\prime}\right)$, where $\bar{G}^{a}$ and $\bar{\Sigma}<$ are related to the Green's function $G^{a}$ and "scattering in" Keldysh self-energy $\Sigma^{<}$through the same gauge transformation as for $\bar{G}^{r}$. The Keldysh self-energies $\Sigma^{<}\left(t, t^{\prime}\right)$ and $\Sigma^{>}\left(t, t^{\prime}\right)$ are related to the retarded and advanced self-energies through

$$
\begin{aligned}
\Sigma^{<}\left(t, t^{\prime}\right)-\Sigma^{>}\left(t, t^{\prime}\right)= & \Sigma^{r}\left(t, t^{\prime}\right)-\Sigma^{a}\left(t, t^{\prime}\right) \\
= & 2 i \sum_{\eta} \Gamma_{\eta} \\
& \times \int \frac{d \epsilon}{2 \pi \hbar}\left[1+n_{\eta}(\epsilon)\right] \\
& \times e^{-(i / \hbar) \int_{t^{\prime}}^{t}\left[\epsilon+V_{\eta} \cos \left(\omega_{0} t^{\prime \prime}\right)\right] d t^{\prime \prime}},
\end{aligned}
$$

the last equality comes from our approximate expression for self-energies $\Sigma^{r}$ and $\Sigma^{a}$. The factor 2 comes from the factor $\left(1-\left\langle n_{d \sigma}\right\rangle\right)^{-1}$ in the Kondo limit which appears because Eq. (3a) is not written in the standard form of the Dyson equation because of the same factor. When Eq. (3a) is rewritten in the standard Dyson equation form, the $\left(1-\left\langle n_{d \sigma}\right\rangle\right)^{-1}$ factor appears in the properly defined retarded self-energy. The same is true also for the advanced self-energy. To determine $\Sigma^{<}$and $\Sigma^{>}$, we assume further that the self-energies have the form

$$
\begin{gathered}
\Sigma^{<}\left(t, t^{\prime}\right)=i \int \frac{d \epsilon}{\Gamma}\left(\sum_{\eta} \Gamma_{\eta} n_{\eta}(\epsilon)\right) \Sigma^{(0)}\left(\epsilon, t, t^{\prime}\right), \\
\Sigma^{>}\left(t, t^{\prime}\right)=-i \int \frac{d \epsilon}{\Gamma}\left(\sum_{\eta} \Gamma_{\eta}\left[1-n_{\eta}(\epsilon)\right]\right) \Sigma^{(0)}\left(\epsilon, t, t^{\prime}\right),
\end{gathered}
$$

where $\Sigma^{(0)}\left(\epsilon, t, t^{\prime}\right)$ is a function which has to be determined. This assumed form has the advantages that (i) it is exact in the equilibrium limit $\mu_{L}=\mu_{R}$ and without ac bias, (ii) it is exact in the noninteracting $(U=0)$ limit under general nonequilibrium situations [7], and (iii) the continuity equation $J_{L}(t)=-J_{R}(t)$ is automatically satisfied in the steady state (no ac bias) limit. With Eqs. (4) 
and (5), we obtain an approximate expression for $\Sigma^{<}\left(t, t^{\prime}\right)$ and the Keldysh Green's function $G^{<}\left(t, t^{\prime}\right)$ can be determined accordingly. After some algebra, we obtain

$$
\begin{aligned}
& J_{\eta}(t)=-\frac{e}{\hbar} \Gamma_{\eta} \int \frac{d \omega}{2 \pi}\left\{2 n_{\eta}(\omega) \operatorname{Im}\left[A_{\eta}(\omega, t)\right]+\Sigma^{<}(\omega)\right. \\
&\left.\times\left|A_{\eta}(\omega, t)\right|^{2}\right\},
\end{aligned}
$$

where [7]

$$
A_{\eta}(\omega, t)=\int_{-\infty}^{t} d t^{\prime} e^{i \omega\left(t-t^{\prime}\right)} e^{(i / \hbar) \int_{t^{\prime}}^{t} d t^{\prime \prime} V_{\eta} \cos \left(\omega_{0} t^{\prime \prime}\right)} G_{d}^{r}\left(t, t^{\prime}\right)
$$

and

$$
\Sigma^{<}(\omega)=\frac{2}{\Gamma}\left(\sum_{\eta} \Gamma_{\eta} n_{\eta}(\hbar \omega)\right)\left(\sum_{\eta^{\prime}} \Gamma_{\eta^{\prime}}\left[1+n_{\eta^{\prime}}(\hbar \omega)\right]\right) .
$$

Equations (3) and (6) constitute a set of equations where the current response of the system to arbitrary external time-dependent voltage fluctuations can be computed when $V_{\eta}\left(V_{0}\right) \cos \left(\omega_{0} t\right)$ is replaced by general $V_{\eta}\left(V_{0}\right)(t)$. Nevertheless, in the following we shall restrict ourselves to harmonic perturbations and to the linear response regime when the time-dependent external fields are weak. For simplicity we shall consider the symmetric case $\Gamma_{L}=\Gamma_{R}$ and shall consider dc bias voltage with $\mu_{L} \geq \mu_{R}$ and ac bias voltage with $V_{L}=V_{\mathrm{ac}}$, $V_{0}=0.5 V_{L}$, and $V_{R}=0$, corresponding to a symmetric structure. In this case it is easy to see that the linear response current has a form

$$
J_{L(R)}^{\mathrm{ac}}(t) \sim-(+) G_{L(R)}\left(\omega_{0}\right) \cos \left[\omega_{0} t-\delta_{L(R)}\left(\omega_{0}\right)\right] V_{\mathrm{ac}},
$$

where the linear response behavior is determined by the ac conductances $G_{\eta}\left(\omega_{0}\right)$ and phase shifts $\delta_{\eta}\left(\omega_{0}\right)$

At equilibrium $\left(\mu_{L}=\mu_{R}\right)$, it is easy to see by symmetry that $J_{L}^{\mathrm{ac}}(t)=-J_{R}^{\mathrm{ac}}(t)$. However, such a symmetry is destroyed once the chemical potentials $\mu_{L}$ and $\mu_{R}$ are not equal. In Fig. 1 we show the equilibrium ac conductance $G_{L}\left(\omega_{0}\right)=G_{R}\left(\omega_{0}\right)=G\left(\omega_{0}\right)$ (solid line) and phase shift $\delta_{L}\left(\omega_{0}\right)=\delta_{R}\left(\omega_{0}\right)=\delta\left(\omega_{0}\right)$ (dashed line) as a function of frequency $\omega_{0}$ for three different values of temperatures, $T=0.5 T_{K}$ (i), $T=4 T_{K}$ (ii), and $T=8 T_{K}$ (iii), computed in our equation of motion approach. The ac conductance $G\left(\omega_{0}\right)$ is measured in units of the zero temperature dc conductance $G(0,0)$, whereas the phase shift $\delta\left(\omega_{0}\right)$ is measured in units of $\pi$. We have chosen parameters $D=100, \Gamma_{L}=\Gamma_{R}=1.0$, and $\epsilon_{0}=-4.5$ in the above calculation. The Kondo temperature $T_{K}$ is equal to 0.0017 using these parameters. The appearance of Kondo resonance [9] at low frequency which vanishes as temperature increases is clear from the figure. Next we consider the ac responses for the out of equilibrium Anderson impurity. In Figs. 2 and 3 we show the ac responses of an out of equilibrium Anderson impurity at fixed temperature $T=T_{K}$ for three diffferent values of voltage differences:

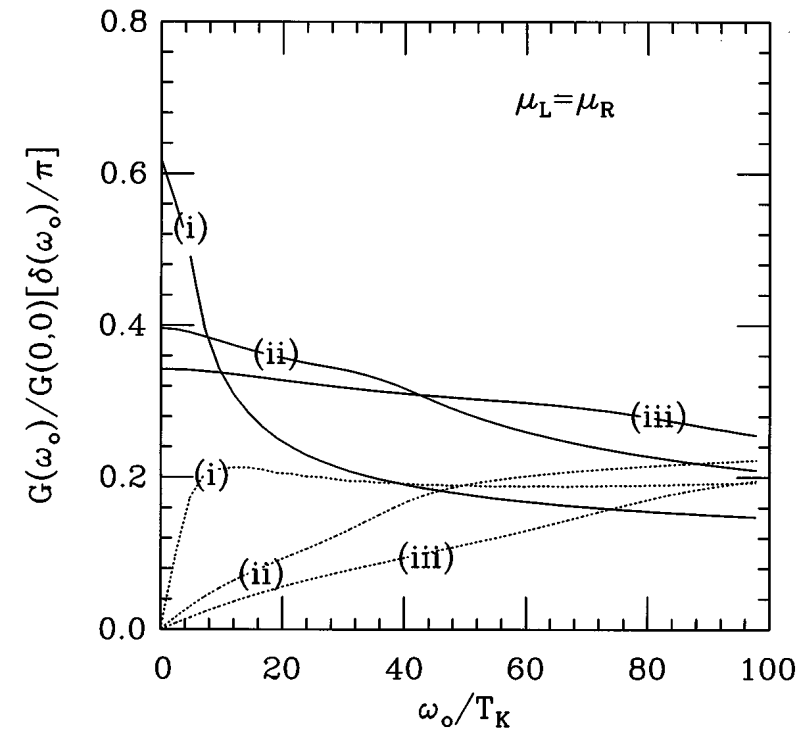

FIG. 1. ac responses for an Anderson impurity at equilibrium $\left(\mu_{L}=\mu_{R}\right)$ for three different values of temperatures $T=$ $0.5 T_{K}$ (i), $T=4 T_{K}$ (ii), and $T=8 T_{K}$ (iii). Solid lines show the ac conductances, whereas dashed lines show the phase shifts.

$\Delta \mu=\mu_{L}-\mu_{R}=2 T_{K}$ (i), $20 T_{K}$ (ii), and $50 T_{K}$ (iii) for the same set of parameters $D, \Gamma_{\eta}$, and $\epsilon_{0}$. The ac conductances (solid lines) and phase shifts (dashed lines) for the left and right currents $J_{L}(t)$ and $J_{R}(t)$ are shown, respectively, in Figs. 2 and 3. The behavior of the left and right current responses are quite different, as can be seen from the figures. For the left current, "shoulder" structures are seen in the conductance $G_{L}\left(\omega_{0}\right)$ at frequencies $\omega_{0} \sim \Delta \mu$, whereas "dips" are found in the right conductance $G_{R}\left(\omega_{0}\right)$ as $\omega_{0}$ increases from zero. The conductance

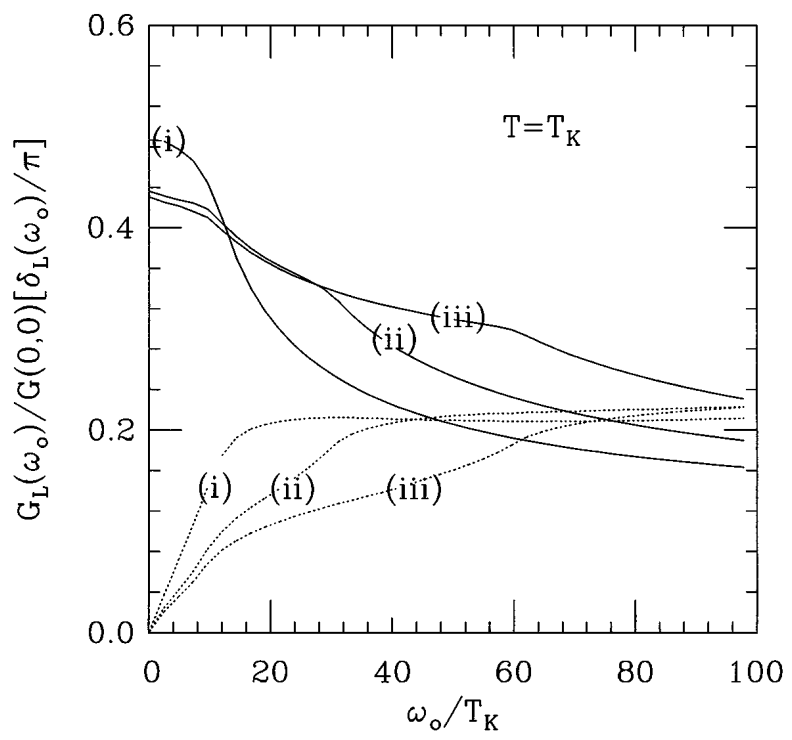

FIG. 2. ac responses for the "left" current $J_{L}(t)$ at fixed temperature $T=T_{K}$ and for three different values of voltage differences $\Delta \mu=2 T_{K}$ (i), $20 T_{K}$ (ii), and $50 T_{K}$ (iii). 


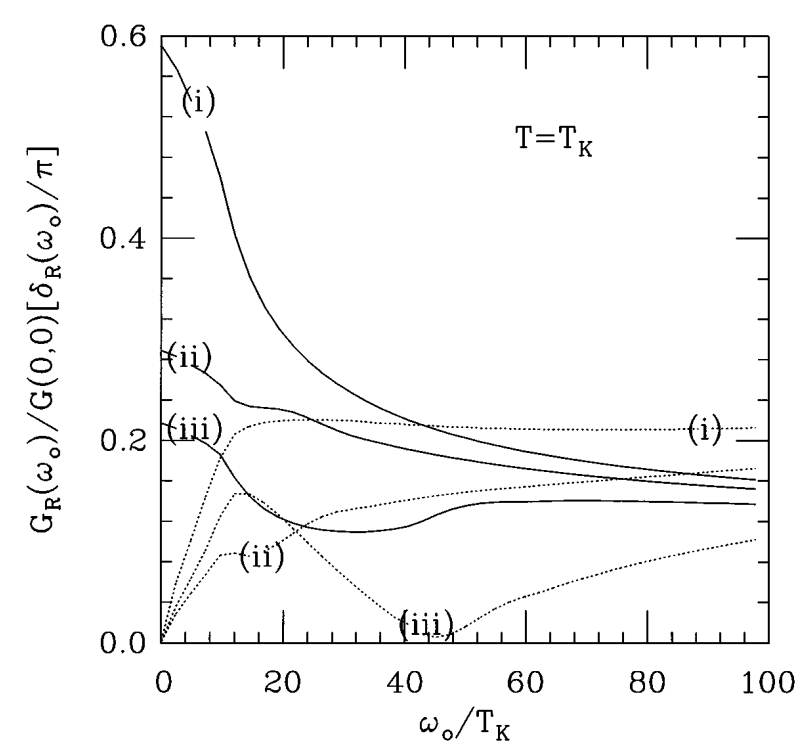

FIG. 3. ac responses for the "right" current $J_{R}(t)$ at fixed temperature $T=T_{K}$ and for three different values of voltage differences $\Delta \mu=2 T_{K}$ (i), $20 T_{K}$ (ii), and $50 T_{K}$ (iii).

starts to rise again at frequencies $\omega_{0} \sim \Delta \mu$. The phase shift of the right current also shows a clear dip at frequencies $\omega_{0} \sim \Delta \mu$. However, such a structure is missing in the left current response. The "magnitude" of the resonant signals were not found to be very strong in our calculation, however, resonant behaviors as $\omega_{0} \sim \Delta \mu$ are clearly observed from both the left and right current responses. We note that the qualitative behaviors of the current responses are insensitive to the particular parameters we choose in producing these results. As temperature rises these structures are gradually washed away. In fact, at $T=8 T_{K}$, we already found that the current responses for all three different values of $\Delta \mu$ 's are very similar to the current response of the $\Delta \mu=0$ case at $T=8 T_{K}$, as shown in Fig. 1. This is hardly surprising since the Kondo temperature and thus the voltage differences $\Delta \mu$ we consider in our calculation are much less than any other microscopic energy scales in our system.

It has to be emphasized that because of the crudeness of the equation of motion approach, our results for the ac response can only be trusted qualitatively. In particular, the precise "magnitude" of the resonances cannot be obtained accurately from our crude approach. A more quantitative analysis of the ac responses in the out of equilibrium Anderson impurity model can be achieved only by using more rigorous methods. For example, Hettler and Schoeller [10] have studied time-dependent perturbations on the Anderson impurity using $1 / N$ expansion where similar resonant effects were discussed. However, the frequency-dependent current responses were not being considered in their paper. In any case, our results suggest clearly that the out of equilibrium Kondo reso- nances in the Anderson impurity model will give rise to interesting observable effects in current responses of the system to ac bias voltage. Because of its simplicity, the equation of motion approach we developed in this paper can be extended quite easily to compute responses of the Anderson impurity to an arbitrary form of external timedependent voltage fluctuations at arbitrary temperature. The main disadvantage of the method is that only qualitative behaviors of the response of Anderson impurity to time-dependent voltage fluctuations can be extracted from the theory.

Summarizing, using a generalized equation of motion approach, we have computed in this paper linear responses of an out of equilibrium Anderson impurity to external ac bias voltage fluctuations. We show that in the low temperature limit the ac current responses provide another important experimental tool where both the equilibrium and out of equilibrium Kondo resonances can be probed. In particular, in the out of equilibrium case, resonant behavior as the ac frequency matches dc voltage bias is shown to exist as a result of "double-peak" structure in the one-electron density of states at the Kondo regime. The predicted resonant behaviors for the left and right currents $J_{L}(t)$ and $J_{R}(t)$ are found to be rather different, a result which has to be tested experimentally.

The author thanks Shechao Feng for many stimulating discussions during his visit to HKUST which lead to this work. This work is supported by UGC Hong Kong, through RGC Grant No. UST636/94P.

[1] P.L. MaEuen et al., Phys. Rev. Lett. 66, 1926 (1991); A. T. Johnson et al., Phys. Rev. Lett. 69, 1592 (1992); U. Meirav, M. Kastner, and S. J. Wind, Phys. Rev. Lett. 65, 771 (1990); D. C. Ralph and R. A. Buhrman, Phys. Rev. Lett. 69, 2118 (1992).

[2] D. C. Ralph and R. A. Buhrman, Phys. Rev. Lett. 72, 3401 (1994).

[3] S. Hershfield, J. H. Davies, and J. W. Wilkins, Phys. Rev. Lett. 67, 3720 (1991).

[4] Y. Meir, N. S. Wingreen, and P. A. Lee, Phys. Rev. Lett. 70, 2601 (1993).

[5] T. K. Ng, Phys. Rev. Lett. 70, 3635 (1993).

[6] M. H. Hettler, J. Kroha, and S. Hershfield, Phys. Rev. Lett. 73, 1967 (1994).

[7] N. S. Wingreen, A.P. Jauho, and Y. Meir, Phys. Rev. B 48, 8487 (1993); A.P. Jauho, N.S. Wingreen, and Y. Meir, Phys. Rev. B 50, 5528 (1994).

[8] Y. Meir, N. S. Wingreen, and P. A. Lee, Phys. Rev. Lett. 66, 3048 (1991).

[9] T. K. Ng and P. A. Lee, Phys. Rev. Lett. 61, 1768 (1988); L. I. Glazman and M. E. Raikh, JETP Lett. 47, 452 (1988).

[10] M. H. Hettler and H. Schoeller, Phys. Rev. Lett. 74, 4907 (1995). 